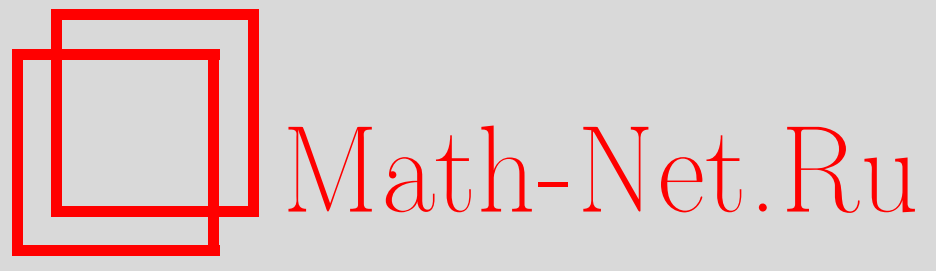

С. С. Марченков, Эквациональное замыкание, Дискрет. матем., 2005, том 17, выпуск 2, 117-126

DOI: https://doi.org/10.4213/dm103

Использование Общероссийского математического портала Math-Net.Ru подразумевает, что вы прочитали и согласны с пользовательским соглашением http://www.mathnet.ru/rus/agreement

Параметры загрузки:

IP : 3.85 .73 .92

26 апреля 2023 г., 12:32:05

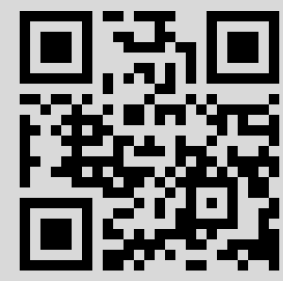


Удк 519.716

\title{
Эквациональное замыкание
}

\author{
() 2005 г. С. С. Марченков
}

\begin{abstract}
На основе исчисления равенств определяется оператор эквационального замыкания. Приводятся примеры эквационально полных систем и эквационально замкнутых классов. Определяется мощность семейства эквационально предполных классов и даются критерии эквациональной полноты. Находятся все эквационально замкнутые классы булевых функций.

Работа выполнена при поддержке Российского фонда фундаментальных исследований, проект 03-01-00783.
\end{abstract}

В теории функций многозначной логики одно из центральных мест принадлежит вопросам классификационного характера. При построении различных классификаций чаще всего используется подход, при котором классификации определяются на основе некоторых операторов замыкания. Самая известная классификация этого типа - классификация на основе оператора (операции) суперпозиции. Для функций двузначной логики (булевых функций) она приводит к счетной решетке замкнутых классов $[26,27,22,12]$. Однако уже для функций трехзначной логики подобная классификация оказывается континуальной [23].

Существует ряд классификаций, которые базируются на операторах замыкания более широких, нежели оператор суперпозиции $[6,3,24,2,19,15-17,9-14,18]$. Все они приводят к конечным классификациям, хотя в основе лежат различные идеи.

Одна из идей, широко представленная в математике, - идея исчисления равенств (тождественных преобразований). На основе этой идеи можно определять и преобразовывать функции, формулы, схемы, программы и другие объекты. Не вдаваясь в многочисленные применения исчисления равенств, укажем лишь на один близкий нам пример - эрбрангеделевское определение частично рекурсивных функций (см., например, [4]).

В настоящей статье мы хотим применить идею исчисления равенств для определения оператора замыкания (эквациональное замыкание) и исследовать основные свойства получающейся при этом классификации.

Введем основные понятия. Пусть $k \geqslant 2, E_{k}=\{0,1, \ldots, k-1\}, P_{k}-$ множество всех функций, определенных на $E_{k}$, то есть всех функций $f$ вида $f: E_{k}^{n} \rightarrow E_{k}$, где $n=1,2, \ldots$ Функщии множества $P_{k}$ носят название функций $k$-значной логики. Для любого $c \in E_{k}$ рассматриваем в $P_{\boldsymbol{k}}$ функцию-константу $c$, зависящую от одной переменной. Если $\boldsymbol{n} \geqslant 1$ и $1 \leqslant i \leqslant n$, то через $e_{i}^{n}\left(x_{1}, \ldots, x_{n}\right)$ обозначаем селекторную функцию, равную переменной $x_{i}$. Считаем известными понятие суперпозиции и связанные с ним понятия замыкания и замкнутого класса (см., например, [21]). Для произвольного множества $Q$ через [Q] обозначаем замыкание множества $Q$ относительно операции суперпозиции. Если $n \geqslant 1$, то $Q^{(n)}$ обозначает множество всех $n$-местных функций из $Q$. 
Будем использовать символы $\varphi_{i}^{(n)}$ в качестве функциональных переменных с областью значений $P_{k}^{(n)}, n=1,2, \ldots$ В случаях, когда это не вызывает недоразумения, у переменных $\varphi_{i}^{(n)}$ опускаем индексы. В качестве индивидуальных переменных используем символы $x, y, z$, возможно, с индексами.

Пусть $Q \subseteq P_{k}$. Будем предполагать, что каждая функщия из $Q$ имеет индивидуальное обозначение. Для обозначения функций из $Q$ используем символ $f$ с индексами (функциональная константа).

Обычным образом по индукции определяем понятие терма над $Q$. Пусть $\varphi$ есть $n$-местная функщиональная переменная, $f-n$-местная функщиональная константа, а $t_{1}, \ldots, t_{n}$ - индивидуальные переменные (не обязательно различные). Тогда выражения $\varphi\left(t_{1}, \ldots, t_{n}\right)$ и $f\left(t_{1}, \ldots, t_{n}\right)$ суть термы над $Q$. Далее, если $t_{1}, \ldots, t_{n}$ суть термы над $Q$ либо символы индивидуальных переменных, а $\varphi$ и $f$ являются соответственно $n$-местной функциональной переменной и $n$-местной функциональной константой, то $\varphi\left(t_{1}, \ldots, t_{n}\right)$ и $f\left(t_{1}, \ldots, t_{n}\right)$ суть термы над $Q$.

Если $t_{1}, t_{2}$ - термы над $Q$, то выражение $t_{1}=t_{2}$ называем равенством над $Q$.

Пусть $t_{1}=t_{2}$ есть равенство над $Q$. Частным случаем равенства $t_{1}=t_{2}$ называем любое равенство вида $t_{1}^{\prime}=t_{2}^{\prime}$, где выражения $t_{1}^{\prime}, t_{2}^{\prime}$ получаются из термов $t_{1}, t_{2}$ подстановкой вместо всех индивидуальных переменных некоторых значений из $E_{k}$. При этом все вхождения одной и той же индивидуальной переменной в термы $t_{1}, t_{2}$ заменяются одним и тем же значением из $E_{k}$.

Пусть $E$ - конечная система равенств над $Q$. Последовательность равенств $T_{1}, \ldots, T_{s}$, не содержащих индивидуальных переменных, называется выводом из системы равенств $E$, если каждое равенство $T_{i}$ этой последовательности удовлетворяет одному из следующих условий:

(1) $T_{i}$ есть частный случай одного из равенств системы $E$,

(2) для некоторого $j<i$ равенство $T_{i}$ получается из равенства $T_{j}$ заменой выражения вида $f\left(a_{1}, \ldots, a_{n}\right)$ значением $a$, где $a_{1}, \ldots, a_{n} \in E_{k}$ и $a=f\left(a_{1}, \ldots, a_{n}\right)$,

(3) для некоторых $j, l<i$ равенство $T_{i}$ получается из равенства $T_{j}$ заменой выражения вида $\varphi\left(a_{1}, \ldots, a_{n}\right)$ значением $a$, где равенство $T_{l}$ имеет вид $\varphi\left(a_{1}, \ldots, a_{n}\right)=a$ или $a=\varphi\left(a_{1}, \ldots, a_{n}\right)$ и $a, a_{1}, \ldots, a_{n} \in E_{k}$.

Равенство $T$, не содержащее индивидуальных переменных, называется выводимым из системы равенств $E$, если существует вывод из системы равенств $E$, который содержит равенство $T$. Система равенств $E$ называется корректной, если из нее невозможно вывести два равенства вида $\varphi\left(a_{1}, \ldots, a_{n}\right)=a, \varphi\left(a_{1}, \ldots, a_{n}\right)=b$, где $a \neq b$ (здесь и далее мы не различаем равенства $\varphi\left(a_{1}, \ldots, a_{n}\right)=a$ и $\left.a=\varphi\left(a_{1}, \ldots, a_{n}\right)\right)$.

Пусть $Q \subseteq P_{k}, E$ - корректная система равенств над $Q, \varphi-n$-местная функциональная переменная, входящая в равенства системы $E$. Говорим, что функция $g\left(x_{1}, \ldots, x_{n}\right) \in P_{k}$ определяется системой равенств $E$ над $Q$, если для любых $a_{1}, \ldots, a_{n} \in E_{k}$ из системы равенств $E$ выводимо равенство вида $\varphi\left(a_{1}, \ldots, a_{n}\right)=a$, где $a=g\left(a_{1}, \ldots, a_{n}\right)$. Множество всех функщй, выводимых из системы равенств $E$, обозначим через $E(Q)$. Положим

$$
\operatorname{Eq}[Q]=\bigcup E(Q)
$$

где объединение берется по всем корректным системам равенств $E$ над $Q$. 
Нетрудно видеть, что Еq является оператором замыкания на множестве $P_{k}$. Иными словами, для любых подмножеств $Q, R$ множества $P_{k}$ выполняются следующие три свойства.

(1) $Q \subseteq \operatorname{Eq}[Q]$.

(2) Если $Q \subseteq R$, то $\mathrm{Eq}[Q] \subseteq \mathrm{Eq}[R]$.

(3) $\mathrm{Eq}[\mathrm{Eq}[Q]]=\operatorname{Eq}[Q]$.

Оператор Eq называем оператором эквационального замыкания.

Легко показать, что $[Q] \subseteq \mathrm{Eq}[Q]$. В самом деле, пусть $f_{0}, f_{1}, \ldots, f_{m} \in Q$ и, например,

$$
f\left(x_{1}, \ldots, x_{n}\right)=f_{0}\left(f_{1}\left(x_{1}^{1}, \ldots, x_{n_{1}}^{1}\right), \ldots, f_{m}\left(x_{1}^{m}, \ldots, x_{n_{m}}^{m}\right)\right) \text {, }
$$

где

$$
\left\{x_{1}, \ldots, x_{n}\right\}=\left\{x_{1}^{1}, \ldots, x_{n_{1}}^{1}, \ldots, x_{1}^{m}, \ldots, x_{n_{m}}^{m}\right\},
$$

а системы корректных равенств $E^{0}, E^{1}, \ldots, E^{m}$ определяют соответственно функции $f_{0}, f_{1}, \ldots, f_{m}$. Предположим далее, что множества функциональных переменных систем равенств $E^{0}, E^{1}, \ldots, E^{m}$ не пересекаются, а функциям $f_{0}, f_{1}, \ldots, f_{m}$ соответствуют функциональные переменные $\varphi_{0}, \varphi_{1}, \ldots, \varphi_{m}$. Если функциональная переменная $\varphi$ не встречается в системах равенств $E^{0}, E^{1}, \ldots, E^{m}$ и система равенств $E$ состоит из всех равенств систем $E^{0}, E^{1}, \ldots, E^{m}$ и равенства

$$
\varphi\left(x_{1}, \ldots, x_{n}\right)=\varphi_{0}\left(\varphi_{1}\left(x_{1}^{1}, \ldots, x_{n_{1}}^{1}\right), \ldots, \varphi_{m}\left(x_{1}^{m}, \ldots, x_{n_{m}}^{m}\right)\right),
$$

то, очевидно, система равенств $E$ является корректной и определяет функцию $f$.

Понятия Еq-полного множества, Еq-замкнутого и Еq-предполного классов представляют собой естественные аналоги соответствующих понятий для оператора замыкания, основанного на суперпозиции.

Предложение 1. При любом $k \geqslant 2$ система $\{0,1, \ldots, k-1\}$ всех функций-констант из $P_{k}$ полна в $P_{k}$.

Доказательство. Пусть $g\left(x_{1}, \ldots, x_{n}\right)$ - произвольная функция из $P_{k}$. Ее можно определить с помощью системы равенств над множеством $\{0,1, \ldots, k-1\}$, которая состоит из всех равенств вида $\varphi\left(a_{1}, \ldots, a_{n}\right)=b$, где $a_{1}, \ldots, a_{n} \in E_{k}$ и $b=g\left(a_{1}, \ldots, a_{n}\right)$.

Следствие 1. Любой Еq-замкнутый класс функций из $P_{k}$, отличный от $P_{k}$, можно расиирить до Еq-предполного в $P_{k}$ класса.

Это следствие есть частный случай хорошо известного общего утверждения из теории множеств и универсальных алгебр (см., например, теорему 5.4 в [5]).

Из полученного следствия вытекает критерий Еq-полноты в $P_{k}$.

Теорема 1. Система функчий $Q \subseteq P_{k}$ Еq-полна в классе $P_{k}$ тогда и только тогда, когда $Q$ иеликом не содержится ни в одном из Еq-предполных в $P_{k}$ классов.

Пусть $\rho\left(x_{1}, \ldots, x_{m}\right)$ - предикат на множестве $E_{k}$, то есть отображение $\rho: E_{k}^{m} \rightarrow\{$ И, Л $\}$, где И, Л - истинностные значения истина и ложь. Говорят, что функция $f\left(x_{1}, \ldots, x_{n}\right) \in P_{k}$ сохраняет предикат $\rho$, если для любых $n$ наборов 
$\left(a_{11}, \ldots, a_{m 1}\right), \ldots,\left(a_{1 n}, \ldots, a_{m n}\right)$, удовлетворяющих предикату $\rho$, набор $\left(f\left(a_{11}, \ldots, a_{1 n}\right)\right.$, $\left.\ldots, f\left(a_{m 1}, \ldots, a_{m n}\right)\right)$ также удовлетворяет предикату $\rho$.

Обозначим через $\operatorname{Pol}(\rho)$ множество всех функций из $\boldsymbol{P}_{\boldsymbol{k}}$, которые сохраняют предикат $\rho$. Известно (см., например, [1]), что для любого предиката $\rho$ множество Pol $(\rho)$ является замкнутым (относительно суперпозиции) классом, содержащим все селекторные функцин.

Напомним [28], что каждый предполный в $P_{k}$ класс определяется (в смысле функтора Pol) предикатом одного из шести семейств $\mathbf{P}, \mathbf{O}, \mathbf{L}, \mathbf{E}, \mathbf{C}, \mathbf{B}$. При этом семейство $\mathbf{P}$ состоит из предикатов, которые являются графиками перестановок на $E_{k}$, разлагающихся в произведение циклов одной и той же простой длины, а все одноместные предикаты семейства $C$ имеют вид $x \in D$, где $\varnothing \neq D \subset E_{k}$.

Следствие 2. Пусть $Q-$ предполный в $P_{k}$ класс, который определяется предикатом одного из семейств О, L, Е, В или неодноместным предикатом семейства С. Тогда $\mathrm{Eq}[Q]=P_{k}$

Доказательство. Как вытекает из [28], предполный класс $Q$ указанного типа содержит все константы $0,1, \ldots, k-1$.

Пусть $\pi$ - перестановка на множестве $E_{k}$, предикат $\left(\pi\left(x_{1}\right)=x_{2}\right)$ - график перестановки $\pi$ и $Q_{\pi}-$ множество всех функций из $P_{k}$, сохраняющих предикат $\pi\left(x_{1}\right)=x_{2}$. Тогда, как известно, множество $Q_{\pi}$ является замкнутым классом и состоит из всех функций $g\left(x_{1}, \ldots, x_{n}\right)$, которые удовлетворяют тождеству

$$
\pi\left(g\left(x_{1}, \ldots, x_{n}\right)\right)=g\left(\pi\left(x_{1}\right), \ldots, \pi\left(x_{n}\right)\right) .
$$

В этом случае функция $g$ называется также самодвойственной относительно перестановки $\pi$.

Предложение 2. Kлассы типа $Q_{\pi}$ Еq-замкнуты.

Доказательство легко следует из хорошо известного факта (см., например, [20]): если функщии $f_{0}, f_{1}, \ldots, f_{m}$ сохраняют предикат $\pi\left(x_{1}\right)=x_{2}$ (самодвойственны относительно перестановки $\pi$ ) и функция $f$ удовлетворяет соотношению (1), то функщия $f$ также сохраняет предикат $\pi\left(x_{1}\right)=x_{2}$.

Следствие 3. Если перестановка л разлагается в произведение чиклов одной и той же простой длины, то класс $Q_{\pi}$ Еq-предполон в $P_{k}$.

Доказательство вытекает из Еq-замкнутости класса $Q_{\pi}$ и его предполноты в $P_{k}$ (см. $[20,28])$.

Обозначим через $S_{k}$ полную симметрическую группу перестановок на множестве $E_{k}$. Если $G$ - подгруппа группы $\mathrm{S}_{k}$, то пусть $Q_{G}$ есть множество всех функций из $P_{k}$, которые сохраняют графики всех перестановок группы $G$ (самодвойственны относительно всех перестановок группы $G$ ).

Следстьие 4. Классы типа $Q_{G}$ Еq-замкнуты.

В качестве важного частного случая, удовлетворяющего следствию 2, отметим класс всех однородный функций из $P_{k}[25,7]-$ функций, самодвойственных относительно любых перестановок группы $\mathbf{S}_{k}$.

Пусть $D \subseteq E_{k}$. Обозначим через $Q_{D}$ множество всех функщий из $P_{k}$, сохраняющих предикат $x \in D$ (сохраняющих множество $D$ ). 
Предложение 3. Для любого подмножества $D$ множества $E_{k}$ класс $Q_{D}$ является Еq-замкнутым. Если, кроме того, $D \neq \varnothing$ и $D \neq E_{k}$, то класс $Q_{D}$ Еq-предполон в $P_{k}$.

Доказательство легко следует из того, что классы $Q_{D}$ замкнуты (относительно суперпозиции) и предполны при $D \neq \varnothing, D \neq E_{k}[20,28]$.

Пусть $m \geqslant 1, Q \subseteq P_{k}^{(m)}$ и $R \subseteq P_{k}$. Говорят, что множество функщий $R$ сохраняет множество функций $Q$, если для любой функции $f\left(x_{1}, \ldots, x_{n}\right) \in R$ и любых функций $g_{1}, \ldots, g_{n} \in Q$ (среди функций $g_{1}, \ldots, g_{n}$ возможны повторения) функция $f\left(g_{1}\left(x_{1}, \ldots, x_{m}\right), \ldots, g_{n}\left(x_{1}, \ldots, x_{m}\right)\right)$ принадлежит множеству $Q$. Множество всех функций из $P_{k}$, сохраняющих множество $Q$, обозначим через $\operatorname{Pol}(Q)$.

Множество $Q m$-местных функций назовем накрывающим, если для любого $n \geqslant 1$ и любого набора $\left(a_{1}, \ldots, a_{n}\right) \in E_{k}^{n}$ найдутся такие функция $g_{1}, \ldots, g_{n} \in Q$ и такой набор $\left(b_{1}, \ldots, b_{m}\right) \in E_{k}^{m}$, что

$$
\left(g_{1}\left(b_{1}, \ldots, b_{m}\right), \ldots, g_{n}\left(b_{1}, \ldots, b_{m}\right)\right)=\left(a_{1}, \ldots, a_{n}\right) .
$$

Предложение 4. Для любого $k \geqslant 2$ и любого накрывающего множества $Q \subseteq P_{k}^{(m)}$ выполняется соотношение $\operatorname{Pol}(Q) \subseteq \mathrm{Eq}[Q]$.

Доказательство. Возьмем произвольную функцию $f\left(x_{1}, \ldots, x_{n}\right) \in \operatorname{Pol}(Q)$ и произвольный набор функций $g_{1}, \ldots, g_{n}$ из $Q$. Согласно определению множества $\operatorname{Pol}(Q)$ функция

$$
g\left(x_{1}, \ldots, x_{m}\right)=f\left(g_{1}\left(x_{1}, \ldots, x_{m}\right), \ldots, g_{n}\left(x_{1}, \ldots, x_{m}\right)\right)
$$

принадлежит множеству $Q$. Следовательно, если для любых $g_{1}, \ldots, g_{n}$ из $Q$ в систему равенств $E$ включить равенство

$$
\varphi\left(g_{1}\left(x_{1}, \ldots, x_{m}\right), \ldots, g_{n}\left(x_{1}, \ldots, x_{m}\right)\right)=g\left(x_{1}, \ldots, x_{m}\right),
$$

то, пользуясь накрывающим свойством множества $Q$, получим, что система равенств $E$ корректно определяет функцию $f$.

Следствие 5. Пусть $R$ - Еq-замкнутый класс функций и множество $R^{(m)}$ является накрываючим. Тогда $R=\operatorname{Pol}\left(R^{(m)}\right)$.

Для доказательства достаточно заметить, что все функции из $R$ сохраняют множество $R^{(m)}$.

Следствие 6. Пусть $Q$ - накрывающее множество, $Q \subseteq P_{k}^{(m)}$. Тогда существует не более одного Еq-замкнутого класса $R$, для которого $R^{(m)}=Q$.

Клоном называется замкнутый класс функщий, содержащий все селекторные функцин.

Следствие 7. Число Еq-замкнутых клонов в $P_{k}$ меньше, чем $2^{l(k)}$, где $l(k)=k^{k^{k}}$.

Доказательство. Нетрудно видеть, что множество $\left\{e_{1}^{k}, \ldots, e_{k}^{k}\right\}$ селекторных функций является накрывающим. В самом деле, если $\left(a_{1}, \ldots, a_{n}\right)$ - произвольный набор из $E_{k}^{n}$, то функции $e_{a_{1}+1}^{k}\left(x_{1}, \ldots, x_{k}\right), \ldots, e_{a_{n}+1}^{k}\left(x_{1}, \ldots, x_{k}\right)$ на наборе $(0,1, \ldots, k-1)$ принимают соответственно значения $a_{1}, \ldots, a_{n}$. Таким образом, у произвольного Еq-замкнутого клона $R$ множество $R^{(k)}$ также будет накрывающим. Остается применить следствие 2 и заметить, что число всех функций $k$-значной логики, зависящих от $k$ переменных, есть $k^{k^{k}}$. 
Для любого $k \geqslant 2$ обозначим через $\mathbf{T}_{k}$ симметрическую полугруппу подстановок на множестве $E_{k}$, рассматриваемую с операцией композиции (суперпозиции). Подполугруппа $T$ полугрупшы $\mathbf{T}_{k}$ называется транзитивной, если для любых двух элементов $a, b \in E_{k}$ в полугрупше $T$ имеется такая подстановка $f$, что $f(a)=b$.

Предложение 5. Пусть $Q \subseteq P_{k}$ u $Q$ челиком не содержится ни в одном из предполных в $P_{k}$ классов вида $Q_{D}$. Тогда множество $[Q]^{(1)}$ образует транзитивную подполугруппу полугруппы $\mathbf{T}_{\boldsymbol{k}}$.

Доказательство. Пусть $|D|=k-1$ и $b \notin D$. Из соотношения $Q \nsubseteq Q_{D}$ следует, что в множество $Q$ входит функция $f_{1}\left(x_{1}, \ldots, x_{n}\right)$, не сохраняющая $D$. Это означает, что существует такой набор $\left(a_{1}, \ldots, a_{n}\right) \in D^{n}$, что $f_{1}\left(a_{1}, \ldots, a_{n}\right)=b$. Отождествляя в функции $f_{1}$ переменные, которым в наборе $\left(a_{1}, \ldots, a_{n}\right)$ соответствуют одинаковые значения, получим функцию $g_{1}$, зависящую не более чем от $k-1$ переменных и не сохраняющую множество $D$. Можно считать, что функция $g_{1}$ зависит ровно от $k-1$ переменных.

Итак, для любого $D \subset E_{k}$, состоящего из $k-1$ элементов, в классе [Q] имеется такая функция $g_{1}\left(x_{1}, \ldots, x_{k-1}\right)$, что $g_{1}\left(a_{1}, \ldots, a_{k-1}\right)=b$, где $D=\left\{a_{1}, \ldots, a_{k-1}\right\}$ и $b \notin D$.

Продолжим этот процесс и рассмотрим множество $D \subset E_{k}$, состоящее из $k-2$ элементов. По условию предложения, в множество $Q$ входит функция $f_{2}$, не сохраняющая множество $D$. Как и выше, отождествлением переменных из функции $f_{2}$ получаем функцию $g_{2}^{\prime}\left(x_{1}, \ldots, x_{k-2}\right)$, также не сохраняющую множество $D$. Пусть $E_{k} \backslash D=$ $\left\{b_{1}, b_{2}\right\}$. Тогда можно подобрать такой набор $\left(a_{1}, \ldots, a_{k-2}\right)$, что $\left\{a_{1}, \ldots, a_{k-2}\right\}=D$ и выполняется одно из равенств $g_{2}^{\prime}\left(a_{1}, \ldots, a_{k-2}\right)=b_{1}, g_{2}^{\prime}\left(a_{1}, \ldots, a_{k-2}\right)=b_{2}$. Если, например, выполняется первое равенство, то выберем в классе $[Q]$ такую функцию $h\left(x_{1}, \ldots, x_{k-1}\right)$, что $h\left(a_{1}, \ldots, a_{k-2}, b_{1}\right)=b_{2}$. Полагая теперь $g_{2}\left(x_{1}, \ldots, x_{k-2}\right)=$ $h\left(x_{1}, \ldots, x_{k-2}, g_{2}^{\prime}\left(x_{1}, \ldots, x_{k-2}\right)\right)$, получим $g_{2}\left(a_{1}, \ldots, a_{k-2}\right)=b_{2}$.

Таким образом, для любого множества $D \subset E_{k}$, содержащего $k-2$ элементов, в классе $[Q]$ имеются такие функции $g_{2}^{1}, g_{2}^{2}$, зависящие от $k-2$ переменных, что

$$
g_{2}^{1}\left(a_{1}, \ldots, a_{k-2}\right)=b_{1}, \quad g_{2}^{2}\left(a_{1}, \ldots, a_{k-2}\right)=b_{2},
$$

где $\left\{a_{1}, \ldots, a_{k-2}\right\}=D$ и $\left\{b_{1}, b_{2}\right\}=E_{k} \backslash D$.

Продолжая по индукщии, предположим, что для любых двух различных элементов $a_{1}, a_{2} \in E_{k}$ в классе $[Q]$ содержатся такие функции $g_{k-2}^{1}\left(x_{1}, x_{2}\right), \ldots, g_{k-2}^{k-2}\left(x_{1}, x_{2}\right)$, что

$$
g_{k-2}^{1}\left(a_{1}, a_{2}\right)=b_{1}, \ldots, g_{k-2}^{k-2}\left(a_{1}, a_{2}\right)=b_{k-2},
$$

где $\left\{b_{1}, \ldots, b_{k-2}\right\}=E_{k} \backslash\left\{a_{1}, a_{2}\right\}$. Рассмотрим множество $D$, состоящее из одного элемента $a$. По условию предложения в множестве $Q$ имеется функция $f_{k-1}$, не сохраняющая множество $D$. Отождествляя в ней все переменные, получим функцию $g_{k-1}^{1}(x) \in[Q]$, для которой $g_{k-1}^{1}(a) \neq a$. Пусть $g_{k-1}^{1}(a)=b_{1}$ и $b_{2}, \ldots, b_{k-1}$ - остальные элементы множества $E_{k} \backslash\left\{a, b_{1}\right\}$. Если $2 \leqslant i \leqslant k-1$, то по предположению индукции в классе [Q] содержится такая функщия $h_{k-2}^{i}\left(x_{1}, x_{2}\right)$, что $h_{k-2}^{i}\left(a, b_{1}\right)=b_{i}$. Положим при $2 \leqslant i \leqslant k-1$

$$
g_{k-1}^{i}(x)=h_{k-2}^{i}\left(x, g_{k-1}^{1}(x)\right) \text {. }
$$

Тогда, очевидно, $g_{k-1}^{i}(a)=b_{i}$, что завершает доказательство предложения.

Теорема 2. При любом $k \geqslant 2$ число Еq-предполных в $P_{k}$ классов конечно. 
Доказательство. Пусть $Q-$ Еq-предполный в $P_{k}$ класс, отличный от Еq-предполных классов вида $Q_{D}$. Согласно предложению 5 , множество $Q^{(1)}$ образует транзитивную подполугруппу полугруппы $\mathbf{T}_{k}$. Тогда $Q^{(1)}$, очевидно, является накрывающим множеством. Применяя следствие 1 из предложения 4, получаем, что $Q=\operatorname{Pol}\left(Q^{(1)}\right)$. Таким образом, в силу следствия 5 число Еq-предполных в $P_{k}$ классов, отличных от классов вида $Q_{D}$, не превосходит числа транзитивных подполугрупп полугруппы $\mathbf{T}_{\boldsymbol{k}}$.

Теорема доказана.

Определим функщию $p(x, y, z)$ (тернарный дискриминатор) соотношениями

$$
p(x, y, z)= \begin{cases}z, & \text { если } x=y \\ x & \text { в противном случае }\end{cases}
$$

Предложение 6. Пусть $k \geqslant 2, m \geqslant 1, Q \subseteq P_{k}^{(m)}$ u множество $Q$ обладает следуюиим свойством: для любых двух неравных элементов $a_{1}, a_{2} \in E_{k}$ найдутся функции $f_{1} \in Q$ и $f_{2} \in Q \cup\left\{e_{1}^{m}, \ldots, e_{m}^{m}\right\}$ такие, что для некоторых $c_{1}, \ldots, c_{m} \in E_{k}$ выполняются соотношения

$$
\begin{gathered}
f_{1}\left(c_{1}, \ldots, c_{m}\right)=a_{1}, \quad f_{2}\left(c_{1}, \ldots, c_{m}\right)=a_{2}, \\
\left(\forall x_{1}\right) \ldots\left(\forall x_{m}\right)\left(f_{1}\left(x_{1}, \ldots, x_{m}\right) \neq f_{2}\left(x_{1}, \ldots, x_{m}\right)\right) .
\end{gathered}
$$

Tогда $p \in \mathrm{Eq}[Q]$.

Доказательство. Образуем систему равенств $E$, определяющую функцию $p$. Для всякой функции $f \in Q$ в систему $E$ включим равенство

$$
\varphi\left(x, x, f\left(y_{1}, \ldots, y_{m}\right)\right)=f\left(y_{1}, \ldots, y_{m}\right) .
$$

Для любых функций $f_{1} \in Q$ и $f_{2} \in Q \cup\left\{e_{1}^{m}, \ldots, e_{m}^{m}\right\}$, которые удовлетворяют соотношению (3), отнесем к системе $E$ равенство

$$
\varphi\left(f_{1}\left(x_{1}, \ldots, x_{m}\right), f_{2}\left(x_{1}, \ldots, x_{m}\right), z\right)=f_{1}\left(x_{1}, \ldots, x_{m}\right) .
$$

При этом если $f_{2}\left(x_{1}, \ldots, x_{m}\right)=e_{i}^{m}\left(x_{1}, \ldots, x_{m}\right)$, то в (5) вместо $f_{2}\left(x_{1}, \ldots, x_{m}\right)$ оставляем только переменную $x_{i}$.

Покажем, что система равенств $E$ корректно определяет функцию $p$. Первое из равенств (2) гарантирует, что каждое значение из $E_{k}$ принимается некоторой функцией $f \in Q$. Поэтому равенства (4) обеспечивают правильное задание функции $p(x, y, z)$ при $x=y$. Далее, согласно выбору функщий $f_{1}, f_{2}$ в равенстве (5) и условию (2) в равенстве (5) для функции $p(x, y, z)$ будут представлены все пары неравных значений $x, y$. Это завершает доказательство предложения.

Теорема 3. Система функиий $Q \subseteq P_{k}$ Еq-полна в классе $P_{k}$ тогда и только тогда, когда она челиком не содержится ни в одном из Еq-предполных классов типов $Q_{D}, Q_{\pi}$, и для некоторого $m \geqslant 1$ множество $(\mathrm{Eq}[Q])^{(m)}$ обладает свойством, приведенным в предложении 6.

Доказательство. Необходимость условий теоремы очевидна. Докажем достаточность. 
В силу предложения 6 функция $p$ принадлежит классу $\mathrm{Eq}[Q]$. Далее, согласно теореме 2, в [8] всякий замкнутый класс $R \subseteq P_{k}$, содержащий функцию $p$, состоит из функций, которые сохраняют либо предикат

$$
(x \in C) \&(\sigma(x)=y),
$$

где $\varnothing \neq C \subseteq E_{k}$ и $\sigma-$ перестановка на $E_{k}$, либо предикат

$$
\left(x \in D_{1}\right) \&\left(y \in D_{2}\right) \text {, }
$$

где $D_{1}, D_{2}$ - непустые подмножества множества $E_{k}$. При этом в случае $R \neq P_{k}$ хотя бы один из сохраняемых предикатов (6), (7) должен быть нетривиальным. Для предиката (6) это означает, что исключается случай, когда $C=E_{k}$ и $\sigma$ - тождественная перестановка. Для предиката (7) исключается случай, когда $D_{1}=D_{2}=E_{k}$.

Предположим, что $\mathrm{Eq}[Q] \neq P_{k}$, и пусть все функции класса $\mathrm{Eq}[Q]$ сохраняют предикат (6). Тогда, в частности, все функции из $\mathrm{Eq}[Q]$ будут сохранять предикат $x \in C$ (см. [1]). Поэтому при $C \neq E_{k}$ получаем включение $\mathrm{Eq}[Q] \subseteq Q_{C}$, что противоречит условию теоремы. Если же $C=E_{k}$, то множество $\mathrm{Eq}[Q]$ состоит из функций, сохраняющих график перестановки $\sigma$. Следовательно, $\mathrm{Eq}[Q] \subseteq Q_{\sigma}$. Если перестановка $\sigma$ не является тождественной, то либо класс $Q_{\sigma}$ Еq-предполон в $P_{k}$ (когда $\sigma$ разлагается в произведение циклов одной и той же простой длины), либо содержится в предполном классе типа $Q_{D}$ или типа $Q_{\pi}$ (см. [28]). Таким образом, вновь получаем противоречие с условием теоремы.

Предположим, что все функции класса $\mathrm{Eq}[Q]$ сохраняют предикат (7), где оба множества $D_{1}, D_{2}$ непусты и хотя бы одно из них отлично от $E_{k}$. Пусть, например, это будет множество $D_{1}$. Тогда все функции из $\mathrm{Eq}[Q]$ сохраняют предикат $x \in D_{1}$. Из этого следует, что $\mathrm{Eq}[Q] \subseteq Q_{D_{1}}$. Противоречие завершает доказательство теоремы.

В заключение найдем все Еq-замкнутые классы булевых функций.

Пусть $T_{0}, T_{1}, S, T_{01}, S_{01}, C_{0}, C_{1}$ суть, соответственно, замкнутые классы всех булевых функций, которые сохраняют константу 0 , сохраняют константу 1 , самодвойственны, сохраняют константы 0 и 1 , самодвойственны и сохраняют константу 0 , равны константе 0 , равны константе 1 . Ниже вместо $\mathrm{Eq}\left[\left\{g_{1}, \ldots, g_{r}\right\}\right]$ пишем $\mathrm{Eq}\left[g_{1}, \ldots, g_{r}\right]$.

Предложение 7. Имеют место равенства

$$
\begin{gathered}
T_{0}=\mathrm{Eq}[0, x], \quad T_{1}=\mathrm{Eq}[1, x], \quad S=\mathrm{Eq}[\bar{x}], \quad T_{01}=\mathrm{Eq}[x \vee y], \\
S_{01}=\mathrm{Eq}[x], \quad C_{0}=\mathrm{Eq}[0], \quad C_{1}=\mathrm{Eq}[1] .
\end{gathered}
$$

Доказательство. Начнем с класса $C_{0}$. Очевидно, что $C_{0} \subseteq \mathrm{Eq}[0]$. Вместе с тем, если функщия $f\left(x_{1}, \ldots, x_{n}\right)$ отлична от константы 0 и представима, например, в форме (1), то функция $f_{0}$ также не равна константе 0 . Итерация этого утверждения позволяет сделать вывод, что в классе Eq[0] нет функций, отличных от константы 0.

Двойственным образом рассматривается класс $C_{1}$.

Перейдем к классу $S_{01}$. Следующие две системы равенств над $\{x\}$ корректно определяют булевы функции $x_{1} x_{2} \vee x_{1} x_{3} \vee x_{2} x_{3}$ и $x_{1} \oplus x_{2} \oplus x_{3}$ :

$$
\left\{\begin{array} { l } 
{ \varphi _ { 1 } ( x _ { 1 } , x _ { 1 } , x _ { 2 } ) = x _ { 1 } , } \\
{ \varphi _ { 1 } ( x _ { 1 } , x _ { 2 } , x _ { 1 } ) = x _ { 1 } , } \\
{ \varphi _ { 1 } ( x _ { 1 } , x _ { 2 } , x _ { 2 } ) = x _ { 2 } , }
\end{array} \quad \left\{\begin{array}{l}
\varphi_{2}\left(x_{1}, x_{1}, x_{2}\right)=x_{2}, \\
\varphi_{2}\left(x_{1}, x_{2}, x_{1}\right)=x_{2}, \\
\varphi_{2}\left(x_{1}, x_{2}, x_{2}\right)=x_{1}
\end{array}\right.\right.
$$


Система функций $\left\{x_{1} x_{2} \vee x_{1} x_{3} \vee x_{2} x_{3}, x_{1} \oplus x_{2} \oplus x_{3}\right\}$.образует базис класса $S_{01}[22,12]$. Поэтому $S_{01} \subseteq \mathrm{Eq}[x]$. Обратное включение вытекает из предложений 2,3 , соотношения $x \in S \cap T_{0}$ и из того, что пересечение Еq-замкнутых классов $S$ и $T_{0}$ есть Еq-замкнутый класс $S_{01}$.

Рассмотрим класс $T_{01}$. Очевидно, что $x \in \mathrm{Eq}[x \vee y]$. Значит, по доказанному $S_{01} \subseteq \mathrm{Eq}[x \vee y]$. Система функций $\{x \vee y\} \cup S_{01}$ полна в классе $T_{01}[22,12]$. Поэтому $T_{01} \subseteq \mathrm{Eq}[x \vee y]$. Обратное включение следует из предложения 3 , соотношения $(x \vee y) \in T_{0} \cap T_{1}$ и из того, что пересечение Еq-замкнутых классов $T_{0}$ и $T_{1}$ есть Еq-замкнутый класс $T_{01}$.

Аналогичными рассуждениями можно показать, что $T_{01}=\mathrm{Eq}[x y]$.

Рассмотрим класс $S$. Очевидно, что $x \in \mathrm{Eq}[\bar{x}]$. Следовательно, по доказанному $S_{01} \subseteq \mathrm{Eq}[\bar{x}]$. Множество функций $\{\bar{x}\} \cup S_{01}$ полно в классе $S[22,12]$. Поэтому $S \subseteq \mathrm{Eq}[\bar{x}]$. Обратное включение вытекает из предложения 2 и самодвойственности функщии $\bar{x}$.

Как установлено выше, $C_{0} \cup S_{01} \subseteq \mathrm{Eq}[0, x]$. Однако множество $C_{0} \cup S_{01}$ полно в классе $T_{0}[22,12]$. Значит, $T_{0} \subseteq \mathrm{Eq}[0, x]$. Обратное включение следует из предложения 3 и принадлежности обеих функций $0, x$ классу $T_{0}$.

Класс $T_{1}$ рассматривается двойственным образом.

Теорема 4. Все непустые Еq-замкнутые классы булевых функций исчерпываются классами $P_{2}, T_{0}, T_{1}, S, T_{01}, S_{01} ; C_{0}, C_{1}$.

Доказательство. Пусть $R$ - произвольный непустой Еq-замкнутый класс булевых функций. Рассмотрим множество $R^{(1)}$. Если $\{0,1\} \subseteq R^{(1)}$, то, согласно предложению 1 , $R=P_{2}$. Если $R^{(1)}=\{0, x\}$, то множество $R^{(1)}$ является накрывающим и $\operatorname{Pol}(\{0, x\})=T_{0}$ (см., например, [12]). Поэтому следствие 5 дает равенство $R=T_{0}$. Аналогично рассматриваются случаи $R^{(1)}=\{1, x\}$ и $R^{(1)}=\{x, \bar{x}\}$, где $\operatorname{Pol}(\{1, x\})=T_{1}$ и $\operatorname{Pol}(\{x, \bar{x}\})=S$ (см. [12]).

Пусть $R^{(1)}=\{x\}$. В силу предложения 7 выполняется включение $S_{01} \subseteq R$. Класс $S_{01}$ содержится только в классах $P_{2}, S, T_{0}, T_{1}, T_{01}[22,12]$. Однако функщии 0,1 и $\bar{x}$ не принадлежат множеству $R^{(1)}$. Поэтому остаются две возможности $-R=T_{01}$ и $R=S_{01}$, которые реализуются согласно предложению 7. Если же $R^{(1)}=\{0\}$ или $R^{(1)}=\{1\}$, то из предложения 7 получаем, что соответственно $C_{0} \subseteq R$ или $C_{1} \subseteq R$. Вместе с тем, любой замкнутый класс булевых функций, отличный от классов $C_{0}$ и $C_{1}$, содержит либо обе константы 0,1 , либо функщию $x$ (см. $[22,12])$. Это завершает доказательство теоремы.

\section{Список литературы}

1. Боднарчук В. Г., Калужнин Л. А., Котов В. Н., Ромов Б. А., Теория Галуа для алгебр Поста. Кибернетика (1969) 3, 1-10; 5, 1-9.

2. Голунков Ю. В., Полнота систем функций в операторных алгоритмах, реализующих функции $k$-значной логики. Вероятностные методы и кибернетика (1980) №17, 23-34.

3. Данильченко А. Ф., О параметрической выразимости функций трехзначной логики. Алгебра $и$ логика (1977) 16, №4, 397-416.

4. Клини С. К., Введение в метаматематику. ИЛ, Москва, 1957.

5. Кон П., Универсальная алгебра. Мир, Москва, 1968.

6. Кузнецов А. В., О средствах для обнаружения невыводимости и невыразимости. В кн.: Логический вывод. Наука, Москва, 1979, с. 5-33.

7. Марченков С. С., Однородные алгебры. Проблемы киберн. (1982) 39, 85-106. 
8. Марченков С. С., Клоновая классификация дуально дискриминаторных алгебр с конечным носителем. Матем. заметки (1997) 61, №3, 359-366.

9. Марченков С. С., $S$-классификация функций многозначной логики. Дискретная математика (1997) 9, №3, 125-152.

10. Марченков С. С., $A$-классификация функций многозначной логики. Докл. РАН (1999) 366, №4, 455-457.

11. Марченков С. С., О выразимости функций многозначной логики в некоторых логикофункциональных языках. Дискретная математика (1999) 11, №4, 110-126.

12. Марченков С. С., Замкнутые классы булевых функций. Физматлит, Москва, 2000.

13. Марченков С. С., S-классификачия функций трехзначной логики. Физматлит, Москва, 2001.

14. Марченков С. С., Операторы замыкания с разветвлением по предикату. Вестник МГУ. Серия 1. Математика, механика (2003) 6, 37-39.

15. Нгуен Ван Хоа, О структуре самодвойственных замкнутых классов трехзначной логики $P_{3}$. Дискретная математика (1992) 4, №4, 82-95.

16. Нгуен Ван Хоа, О семействах замкнутых классов $k$-значной логики, сохраняемых всеми автоморфизмами. Дискретная математика (1993) 5, №4, 87-108.

17. Нгуен Ван Хоа, О замкнутых классах $k$-значной логики, самодвойственных относительно транзитивных групп. Дискретная математика (1998) 8, №1, 129-156.

18. Соловьев В. Д., Замкнутые классы в $k$-значной логике с операцией разветвления по предикату. Дискретная математика (1990) 2, №4, 19-25.

19. Тайманов В. А., О функциональных системах $k$-значной логики с операциями программного типа. Докл. АН СССР (1983) 268, №6, 1307-1310.

20. Яблонский С. В., Функциональные построения в $k$-значной логике. Tpуды Матем. ин-та AH CCCP (1958) 51, 5-142.

21. Яблонский С. В., Введение в дискретную математику. Наука, Москва, 1986.

22. Яблонский С. В., Гаврилов Г. П., Кудрявцев В. Б., Функчии алгебры логики и классы Поста. Наука, Москва, 1966.

23. Янов Ю. И., Мучник А. А., О существовании $k$-значных замкнутых классов, не имеющих конечного базиса. Докл. АН СССР (1959) 127, №1, 44-46.

24. Barris S., Willard R., Finitely many primitive positive clones. Proc. Amer. Math. Soc. (1987) 101, №3, 427-430.

25. Marczewski E., Homogeneous operations and homogeneous algebras. Fund. Math. (1964) 56, №2, 81-103.

26. Post E. L., Introduction to a general theory of elementary propositions. Amer. J. Math. (1921) 43, 163-185.

27. Post E. L., The two-valued iterative systems of mathematical logic. Princeton Univ. Press, Princeton, NJ, 1941.

28. Rosenberg I. G., Über die funktionale Vollständigkeit in den mehrwertigen Logiken. Rozpravy Českosl. Acad. Věd., Ř. Mat. Př́r. Věd. (1970) 80, №4, 3-93. 